\section{Artículo de Reflexión}

Cómo citar: C. Barón y E. Caicedo "Transformación digital, un desafío en la educación superior". Inventum, Vol 16 , No. 30 , pp. 3 - 11 doi: $10.26620 /$ uniminuto.inventum.16.30.2021.3-11

Editorial: Corporación Universitaria Minuto de Dios - UNIMINUTO.

ISSN: $1909-2520$

elSSN: $2590-8219$

Fecha de recibido: Enero 10 de 2021 Fecha de aprobado: Febrero 10 de 2021 Fecha de publicación: Marzo 10 de 2021

Conflicto de intereses: los autores han declarado que no existen intereses en competencia.

\title{
Transformación digital, un desafío en la educación superior
}

\author{
Digital transformation, \\ a challenge in higher education.
}

\author{
Transformação digital, \\ um desafio na educação superior
}

\begin{abstract}
Resumen
El progreso hacia la transformación digital de la educación, ha sido una de las aristas de la crisis educativa vivida en América Latina, cuya realidad se hizo más evidente con la abrupta situación de pandemia donde las condiciones de desigualdad y pobreza, cobran un mayor impacto nocivo en las clases sociales menos favorecidas, siendo flagrantemente vulnerado el derecho humano a la educación. En el caso de la educación superior esta problemática se agudiza con el sesgo de intereses particulares, una gobernanza tradicionalista, currículos rígidos, docentes sin o poca formación sobre pedagogías con uso de la tecnología y estudiantes con mínimas o nulas oportunidades de acceso a un computador y a internet. Como conclusión podemos indicar que el aporte que hace los profesionales de las ciencias computacionales y la ingeniería es abismal, pero ningún esfuerzo será suficiente sino se permite el acceso a los estudiantes de forma equitativa a las tecnologías de la información y las comunicaciones, declarando esto como una violación a los derechos constitucionales de primera generación como la igualdad y otros como el acceso a una educación de calidad. Ante este panorama, ¿la transformación digital respaldará la educación en ingeniería frente a pandemia y post-pandemia?
\end{abstract}

Palabras clave: Transformación digital, educación, pandemia, post-pandemia, herramientas tecnológicas.

Cristina Yanneth Barón Hernández cristinabaron@gmail.com https: / / orcid.org/0000-0002-1622-9753 Magister en Derecho

Universidad Santo Tomás sede Bogotá Colombia

Edisson Caicedo Rojas ercaicedo@unisangil.edu.co https://orcid.org/0000-0001-9702-0144 Fundación Universitaria de San Gil UNISANGIL Chiquinquirá

Colombia.

\section{Abstract}

The parsimonious progress towards the digital transformation of education has been one of the edges of the educational crisis experienced in Latin America, whose reality became more evident with the abrupt pandemic situation where the conditions of inequality and poverty take on a greater impact harmful to the less favored social classes, the human right to education being flagrantly violated. In the case of higher education, this problem is exacerbated by the bias of particular interests, a traditionalist governance, rigid curricula, teachers with little or no training in pedagogies with the use of technology, and students with minimal or no opportunities to access a computer. Internet. As a conclusion we can indicate that the contribution made by professionals in computer science and engineering 
is abysmal, but no effort will be enough if students are not allowed access to information and communication technologies in an equitable way, declaring this as a violation of first generation constitutional rights such as equality and others such as access to quality education. Against this background, will the digital transformation be able to support universities in the resilient action they must adopt to safeguard engineering education or will it increase the gap during pandemic and post-pandemic?

Keywords: Digital transformation, education, pandemic, post-pandemic, Technological tools.

\section{Resumo}

O avanço na transformação digital da educação tem sido uma das pontas da crise educacional vivida na América Latina, cuja realidade se tornou mais evidente com a abrupta situação de pandemia em que as condições de desigualdade e pobreza têm maior impacto prejudicial. classes sociais, o direito humano à educação sendo flagrantemente violado. No caso do ensino superior, esse problema é agravado pelo viés de interesses particulares, uma governança tradicionalista, currículos rígidos, professores com pouca ou nenhuma formação em pedagogias com uso de tecnologia e alunos com oportunidades mínimas ou nenhuma de acesso já a um computador Internet. Em conclusão, podemos indicar que a contribuição dos profissionais da informática e da engenharia é abismal, mas nenhum esforço será suficiente se os alunos não tiverem igual acesso às tecnologias de informação e comunicação, declarando isso uma violação dos direitos constitucionais de primeira geração. como a igualdade e outros como o acesso à educação de qualidade. Nesse contexto, a transformação digital apoiará a educação em engenharia em face da pandemia e pós-pandemia?

Palavras-chave: Transformação digital, educação, pandemia, pós-pandemia, ferramentas tecnológicas. 


\section{I.INTRODUCCIÓN}

El confinamiento vigente ha permitido abrir contacto con el mundo entero a través de la virtualidad, cambiando la manera de vivir, la interacción social y cultural se hace cercana, emergen con más fuerza nuevas modalidades de trabajo, las relaciones comerciales electrónicas se incrementan y, por supuesto la educación superior debe estar a la vanguardia ofreciendo una formación que prepare al futuro profesional. De este modo, la agilidad de la transformación digital, robótica, inteligencia artificial y la automatización, exige de un aprendizaje continuo de constante actualización acoplada a la cuarta revolución industrial.

Repensar las políticas educativas y modificar el paradigma que las decisiones se toman arriba de forma masificada desconociendo la realidad de la gran diversidad de poblaciones con necesidades diversas, puede ser atenuado con una mayor participación activa de todos los sectores que pueden ser fácilmente convocados a través de las herramientas digitales.

De no apoyar a las Instituciones de Educación Superior hacia la transformación digital será inevitable un freno en el aprendizaje puesto que las soluciones para el control de la pandemia no son cercanas y las opciones de regresar a la normalidad están lejanas; de este modo, las herramientas digitales son factor decisivo en aportar a la calidad y cobertura de la educación.

\section{DESARROLLO DE CONTENIDOS}

\section{A. Definiendo la Transformación Digital}

La transformación digital incluye una evolución de pensamiento, usos, costumbres del ser humano, que van a impactar en las empresas al requerir de diversos factores como por ejemplo líderes con una visión futurista y un equipo de colaboradores investidos de competencias apropiadas a una nueva estructura organizacional, estandarte competitivo se basará en la tecnología.

Al respecto la Organización para la Cooperación y el Desarrollo Económico [1] ha manifestado:

(...) la transformación digital no solo se refiere a adoptar tecnologías digitales; también se relaciona con la transformación dentro de la sociedad y del mundo empresarial requerida para convertir a la nueva tecnología en una oportunidad económica y social. Eso requiere inversiones que complementen la tecnología en sí, en competencias, en cambio organizacional, en procesos y modelos de negocios nuevos, así como en los activos intelectuales que ayuden a crear valor a partir de las nuevas tecnologías.

Otros autores [2] consideran "un proceso necesario de profundo cambio tecnológico y cultural que toda organización debe poner en marcha para estar a la altura "de sus clientes digitales".

Es decir, la transformación digital implica a todos los integrantes de la organización una modificación en sus estructuras mentales, la adquisición de una cultura evolucionada, líderes visionarios, equipos de trabajo con competencias digitales, permitiendo adaptarse de manera flexible, rápida e innovadora; contrario sensu quedarían empresas paquidérmicas, estacionadas en su zona de confort con modelos de gestión ausentes de ventajas competitivas en el mercado en un mundo globalizado.

\section{B. Las Ciencias Computacionales y su aporte a la transformación digital}

Las Ciencias Computacionales o Ciencias Formales son la denominación atribuida al uso de las bases teóricas de la informática y la computación mediante el uso de algoritmos que permiten transformar la información a través de un proceso cíclico compuesto de: análisis, diseño, implementación y pruebas; en términos más simples podríamos aseverar que estas ciencias permiten resolver problemas a través de algoritmos definidos de acuerdo a la problemática presentada [3].

En el mundo, las ciencias computacionales se dividen en diversas profesiones que van desde la ingeniería de software, ingeniería informática entre otras. Nuestro país es único, dado que tenemos como pregrado la ingeniería de sistemas, profesión que abarca temas tales como el desarrollo de aplicaciones (software), arquitectura de software, auditoria de sistemas entre otras disciplinas, de ahí radica su importancia y aporte en la evolución tecnológica.

Desde su concepción, el término de ingeniero proviene del latín ingenium equivalente a producir, de ahí que los ingenieros de sistemas generen soluciones a problemas de la vida cotidiana abstrayendo mediante un software el comportamiento real, cuya definición más cercana desde la arista 
de los sistemas es: tomar las cosas del mundo real y transformarlas en código de programación que a través de un entorno integrado de desarrollo generando un programa [4].

Aportes significativos de las ciencias computacionales y la ingeniería son no cuantificables, pero podemos destacar:

1. Charles Babbage: científico y matemático británico quien desarrollo una calculadora mecánica que permite calcular tablas de funciones numéricas, considerándose este avance como la primera computadora creada por el hombre.

2. Ada Lovelace, quien en conjunto con Charles Babbage construyeron la maquina analítica o calculadora de uso general, además de colaborar con este gran avance para la humanidad es considerada como la madre de la programación informática al desarrollar el primer algoritmo procesado por una máquina, lo que hoy podemos considerar como sistemas operativos.

3. Otro aporte de las ciencias computacionales e influyente, aunque desconocido por una gran parte de la sociedad fue introducido por el matemático e informático Alan Turing quien gracias al desarrollo de la maquina enigma o máquina de Turing permitió inclinar la balanza para los aliados, descifrando los códigos de las fuerzas nazis lo que llevaría a culminar la segunda guerra mundial y el fin del régimen de Adolf Hitler.

4. Desde décadas predecesoras, en la evolución del mundo los sistemas han jugado un papel preponderante en el desarrollo de la humanidad, tiempo atrás no se concebía la idea de tener un computador porque era un privilegio de los profesionales en el área, hoy encontramos equipos informáticos en el hogar y es fundamental en el desarrollo de profesiones como la medicina, este es uno de los grandes aportes, impulsado por Steve Paul Jobs, quien antes de fundar Apple, ideo en su ingeniosa mente la posibilidad de que cada persona tuviese una computadora, término que conocemos hoy en día como computación personal y de donde deriva el acrónimo de PC (personal computer).

5. Bill Gates, fundador de la empresa Microsoft, creador del sistema operativo mayormente usado en Latino América (Windows) y quien ha revolucionado la forma de usar los computadores, dado que ofrece un sinnúmero de herramientas ofimáticas tendientes a mejorar cada tarea que se desarrolla en oficinas, hogares y demás lugares donde se ha implementado.

6 Estableciendo una cronología para los aportes de las ciencias computacionales, en la computación moderna surgen herramientas tecnológicas o aplicaciones informáticas que en sus inicios se concibieron como un método de comunicación y de acercamiento entre las personas, las denominadas redes sociales se convirtieron en las aplicaciones con mayor número de usuarios en todo el mundo, incluso su poder ha trascendido de tener contacto con celebridades y personajes de la vida pública hasta influir en decisiones políticas de relevancia mundial. Los creadores de las principales redes sociales como facebook y whatt sapp, no dimensionaron su poder al concebir esta idea, puesto que el primero idealizó el asunto como una agenda inteligente para saber quién está en línea y así poder charlar [5]; y el otro, diseñó su red como una herramienta para conocer y saber diferentes aspectos de las personas vinculadas como amigos.

Estas dos herramientas tecnológicas generaron una gran aceptación en la comunidad, al punto de incrementar las ganancias lucrativas para sus creadores, pero la situación desencadenada por el virus que afecta a la población mundial cambió su uso, paso de ser solo como un medio para conocer y charlar con amigos a convertirse en una de las principales herramientas para docentes y estudiantes en el desarrollo de sus clases mediadas por las tecnologías de la información y las comunicaciones.

Otras herramientas tecnológicas que han sido de gran utilidad para la triada de formación académica (docentes, educandos y padres de familia) son: Skype, Telegram, Zoom y Meet, plataformas que permiten encuentros sincrónicos para que los docentes trasmitan sus conocimientos a los estudiantes y estos puedan participar activamente en su proceso de formación en diferentes áreas y niveles.

Además de las redes sociales usadas para la educación, existen herramientas que tanto instituciones de educación superior como colegios de educación secundaria han implementado, una de ellas es Moodle, la cual es una plataforma de código abierto que permite la creación y administración de contenidos a través de una pagina web, donde los docentes suben material, crean exámenes y muchas más actividades usadas para la enseñanza. 
Otras aplicaciones web que ha tomado un gran renombre son Google Classroom y Edmodo, herramientas que le permiten al docente generar contenidos dinámicos para sus estudiantes, planeando clases, actividades y eventos que son notificados a los estudiantes oportunamente para participar.

Estas, al igual que un sinnúmero de herramientas disponibles en la red, permiten a los docentes ejercer su actividad de educación, pero no podemos desconocer los problemas que esto ha desencadenado, no es un secreto que tanto docentes como estudiantes e instituciones educativas no dimensionaban una situación como la que atraviesa el mundo, exhibiendo los siguientes problemas:

- Ignorancia digital: término que agrupa a todas las personas que carecen de ese dominio de las tecnologías de la información y las comunicaciones, tanto docentes como estudiantes han evidenciado dificultades en el uso de las redes sociales y aplicaciones para adelantar sus clases, esto se deriva de diversos factores entre los que destacamos el difícil acceso a la tecnología en Colombia, la deficiencia en la formación en estas áreas y la poca inversión por parte del estado en infraestructura tecnológica, donde en pleno siglo XXI existen poblaciones sin cobertura de internet.

- Deficiente infraestructura tecnológica: pese a no contar con cifras exactas que demuestren la deficiente cobertura de internet y herramientas (computadores, tabletas o smartphone) podemos inferir que aproximadamente el $50 \%$ de los colombianos (25 millones de acuerdo al censo poblacional del DANE) cuentan con una conexión a internet estable, cifras que denotan esa gran brecha entre los habitantes del territorio nacional [6] por otra parte en el boletín técnico del Departamento Nacional de Estadísticas DANE en el año 2018 apenas 41.6\% los hogares colombianos posee un computador, herramienta fundamental para el acceso a las clases virtuales que se deben realizar debido al confinamiento decretado por el gobierno nacional [7].

Esto sin tener en cuenta los sectores rurales, quienes son los mayormente afectados en toda esta situación, debido a que muchas zonas del país no cuentan con una señal estable de telefonía móvil.

\section{La transformación digital en la educación superior}

A partir del concepto de transformación digital al ser transportado a las Universidades y agregando el ingrediente de pandemia VICA (Volatilidad, Incertidumbre, Complejidad, Ambigüedad) que, de manera disruptiva y vertiginosa aceleró este cambio, presiona a las instituciones a repensar en un modelo organizacional apropiado para enfrentar la pandemia y post-pandemia, esto es, una reformulación de los paradigmas existentes en todos los niveles internos y externos.

A nivel interno la Universidad como organización requiere una transformación digital de todos sus procesos estratégicos, misionales y de apoyo. Por ejemplo, en los procesos de apoyo sería menos complejo y haría confiable los datos de trayectoria académica, diplomas del estudiante si se hace uso del blockchain automatizando su información. Al respecto:

En teoría, el blockchain permitiría acreditar los elementos de un currículo elaborado por el usuario, impidiendo la manipulación o alteración de datos, diseminados través de un sistema distribuido sin guardar los datos en un centro sujeto a ataques o violaciones de su integridad [8].

Por supuesto, esta sería una forma de tener certeza de la documentación al ser presentada en cualquier institución educativa nacional o extranjera, pero requiere por supuesto la implementación y nuevamente vuelve la premisa evolución del pensamiento; si bien no garantizaría las competencias adquiridas, si facilitaría la movilidad en la continuidad de formación académica y actividad laboral en cualquier lugar del mundo.

Resulta relevante examinar algunos aspectos; como fue mencionado la transformación digital requiere cambios no solamente al interior de la Universidad sino también obliga a agentes externos; en otros términos, la clase dirigente del país encargada de la formulación de políticas públicas, propuestas normativas, asignación de presupuesto, estrategias y todo tipo de decisiones en torno a la educación superior con miras a la transformación digital requieren de expertos en contacto directo de las problemáticas con propuestas orientadoras del futuro.

Es conveniente tener a la academia más de cerca con la socialización de sus resultados de 
investigación, permitiendo así mayor interacción universidad - Estado y, en consecuencia se efectuarían disposiciones estatales más acertadas a la necesidad social, política, ambiental, cultural, étnica, religiosa, económica de cada zona colombiana con pertinencia educativa, aprovechamiento de las nuevas tecnologías como instrumento de aprendizaje, extendiendo la cobertura con calidad, preparando educandos y docentes con las competencias necesarias en la nueva era.

Finiquitando este aspecto, un mayor número de universidades deben propender por participar más de cerca en la formulación de políticas públicas educativas a partir de la comunicación a los mandos estatales de las investigaciones en torno a esta transformación, con los efectos favorables de innovar con base en las tecnologías, entregando argumentos sólidos para la toma de decisiones.

Por otro lado, el pensamiento tradicional de los líderes de las Universidades trunca el progreso, citando a Tom Jaap este tipo de pensamiento:

“(...) estimula las organizaciones a continuar basando el aprendizaje y desarrollo en el convencimiento del "síndrome del currículo del tigre de la sabiduría". Este síndrome se enfatiza que se deben aprender y obtener habilidades del pasado. Sin embargo, por mucho que valoremos éste, existe la urgente necesidad de pensar en hoy y en el futuro." [9]

En este sentido, los modelos de organización de las universidades para afrontar la pandemia y post-pandemia, les incumbe hacer una reformulación desde sus cimientos; esto es, tener líderes visionarios, con la capacidad de adaptarse rápidamente al cambio, conscientes de lo conveniente que resulta para la educación asumir la Cuarta Revolución colocando al estudiante en el centro del aprendizaje, capacitando en conocimientos y habilidades a los docentes, personal administrativo, asistencial y de apoyo, generando por demás una cultura en torno a la TD, dejando de lado el copiar sistemas que pueden ser desarrollados con éxito en otros países o regiones, pero diferentes a la caracterización propia.

De otra parte, “la base del liderazgo eficaz está en analizar cuidadosamente la misión de la organización, definirla y fijarla de manera clara y visible." [10]

Las instituciones instan líderes ejecutores de una gobernanza horizontal, participativa basada en la cooperación, colaboración, con acercamiento a la comunidad universitaria, "en el proceso de gobernanza intervienen varios actores, gobierno, organizaciones de la sociedad civil y empresas, generando un gobierno de redes de interacción entre el sector público, privado y civil. [11]. Entre sus prioridades está la atención a sus particulares necesidades, orientando sus objetivos estratégicos al efectivo cumplimiento de las metas y fortaleciendo alianzas con todos los actores intervinientes.

Los mismos autores exponen la concepción de gobernanza universitaria parafraseando "se relaciona a los procedimientos que posibilitan la interacción horizontal de diferentes actores públicos y privados. Dinámica necesaria en el ámbito de la educación superior para viabilizar los acuerdos sociales y cumplir la misión de la universidad como tal" [12].

El líder se caracteriza adicionalmente por el conocimiento propicio a dar impulso con cambios sustanciales en la enseñanza - aprendizaje, conjuntamente con todos los actores involucrados y los procesos derivados, asentándose en la transformación digital como estrategia innovadora en la post-pandemia, previniendo el asumir el uso de la TD solo en calidad de solución temporal por el aislamiento.

La UNESCO se encuentra realizando una iniciativa con visión a 2050 denominada los futuros de la educación, "aprender a transformarse se propone reexaminar y replantear la manera en que la educación y el conocimiento pueden contribuir al bien común mundial", donde tiene participación la población mundial a través de su página web con diversas formas.

La manera de comunicación utilizando canales digitales permite un flujo de información constante beneficiosa en la gestión de las organizaciones por la impronta de celeridad, abriendo a su paso un canal de comunicación directo, transparente, amigable con el cliente interno y externo, posibilidades de incrementar alianzas con otras IES o entidades públicas y privadas, estrategia imperante en el carácter resiliente en post-pandemia.

Ahora bien, haciendo un traslado al proceso de enseñanza - aprendizaje, los currículos rígidos centrados exclusivamente en estrategias pedagógicas con un pensamiento anclado a que la educación presencial es insuperable en calidad, exige que este paradigma sea derrocado del 
pensamiento de la comunidad educativa y se dé un viraje hacia una visión más amplia que rompa esos límites y permita avanzar a la educación superior en entornos flexibles, híbridos entre la presencialidad y la virtualidad.

Hay que romper el esquema mental de que se obtiene un mayor aprendizaje si solamente se imparte clase en el aula física, debe avanzarse hacia una mixtura de herramientas digitales y por ende la transformación del modelo educativo, que permitirá el logro del fin último que es el aprendizaje del estudiante.

Diseñar instituciones que puedan tener una perspectiva de cambio inmediato preparados para el futuro, con un sistema robusto que pueda dar respuesta inmediata y eficiente a eventualidades que circunscriban la presencialidad. Las medidas asumidas en los últimos meses fueron construidas sobre la marcha y las Instituciones de Educación Superior procuraron hacer lo mejor posible frente a la emergente situación donde no se estaba preparado a la necesidad de una este avance.

Por supuesto que la calidad de la educación no la da únicamente hacer uso de unas herramientas digitales, pero si se constituye en un gran aporte a unas mejores prácticas pedagógicas que hace eficaz el aprendizaje. Si bien las IES a causa de la pandemia se vieron obligadas a un plan de choque impartiendo educación remota, no significa con ello que se tenga ya consolidada una transformación digital.

La triada Estado - Empresa -Universidad entra a respaldar todo el sistema educativo alineando todos los aliados posibles en la consecución de atenuar el abrupto cambio de la educación presencial a la remota y, por ende, priorizar la creación e implementación de unos renovados modelos pedagógicos con base en las nuevas tecnologías, procurando minimizar las brechas digitales, garantizando el acceso a equipos de cómputo e internet a la población desfavorecida económicamente o ubicada en zonas urbanas o rurales donde aún no llega la tecnología, caso este último donde tiene cabida la radio comunitaria o la televisión institucional.

Cabe agregar que, al mencionar brecha digital debe verse en un concepto más amplio a acceso y cobertura a las TIC's, sino abarca la diferencia entre las empresas que han aprendido el verdadero valor de las nuevas tecnologías digitales y su impulso dentro de la organización y entre las que omitieron el dominio digital [13].

Ante lo expuesto, la labor docente también debe evolucionar y revolucionar el pensamiento estático, repetitivo a uno holístico, analítico con el uso de nuevas metodologías dinámicas de enseñanza las cuales dominen las competencias digitales que permitan el uso de instrumentos tecnológicos, junto con una formación didáctica y pedagógica para saber dar uso a esa tecnología [13].

El desarrollo de competencias digitales docentes considera: "Desde esta perspectiva, la formación del profesorado en TIC es una de las áreas prioritarias en la actual sociedad del conocimiento, al desempeñar un papel muy importante en las grandes líneas en las que se enmarcarán nuestros sistemas educativos en este nuevo siglo, que se centrarán en la innovación, la globalización, la ruptura de las fronteras culturales y lingüísticas, la movilidad virtual de los estudiantes, la emigración y la formación continua [14]. La interacción docente estudiante con la aprehensión de un innovador modelo de enseñanza-aprendizaje va dar un resignificado a la educación.

La transformación digital es dar un paso delante de la mano con las ciencias computacionales, esto significa que no debe eliminarse todo el modelo educativo sino mejorarlo, flexibilizarlo, ampliando el criterio a un sistema híbrido de presencialidad conjugándolo con el uso de la tecnología.

\section{CONCLUSIONES}

La respuesta al interrogante inicial es que indiscutiblemente la transformación digital es un eje sobre el cual las universidades y puntualmente la educación en la ingeniería deben dar un cambio de paradigma frente al reto de sobreponerse a la crisis producida por la pandemia y el reto de afrontar la post-pandemia; si bien en Colombia la disponibilidad, accesibilidad, aceptabilidad y disponibilidad de la educación ya venía con graves afectaciones que, seguramente de no tomarse medidas adecuadas se tendrá un retroceso y la calidad disminuirá, donde esta última aseveración tendrá respuesta en estudios futuros de cómo fue afectada la calidad de la educación con las medidas de emergencia donde cada institución y docente procuró salir avante. 
La Cuarta Revolución Industrial tiene un efecto transversal en todos los sectores, donde la educación tiene un papel preponderante y junto con sus instituciones su actuar será con un enfoque resiliente e innovador para hacer frente a la post-pandemia.

Por su parte, la TD en los procesos de enseñanza - aprendizaje debe ser insertada a la formación el uso de la tecnología, lo cual no quiere decir que el docente deba dejar de lado los avances hasta ahora llevados en la presencialidad, sino tener una modificación conceptual en nuevas maneras de educar integrando herramientas dadas por la tecnología como estrategias pedagógicas. Lo aquí planteado es un sistema híbrido no solamente en post-pandemia, sino la inserción de los avances tecnológicos revolucionando la experiencia de aprender combinados con la actividad en el aula, abriendo espacios más accesibles de interacción con diversidad de universidades dentro y fuera del país.

La capacitación a docentes en el adecuado uso de las tecnologías debe ser de manera permanente pues inevitablemente se va a tener un retroceso de quedarnos en la educación presencial sin el uso de las TIC y, por otra parte, su uso ausente de la implementación mediante estrategias pedagógicas, generaría otro inconveniente y es el no cumplimiento del fin de la educación que es el aprender. Se tendría un retroceso pues se olvidaría esos conocimientos compartidos generando unos vacíos en educando, que podrán ser medidos en investigaciones ulteriores.

Hay que repensar la educación, pues la situación de salud mundial ralentiza inevitablemente los procesos educativos. La concientización de la mejoría de la calidad y cobertura de la educación superior con el buen uso de las TIC's, permitirá que el uso de las mismas no sea tomado como un remedio a corto plaza en aras de enfrentar la pandemia, sino sea visto como innovación en la forma de enseñar y aprender en post-pandemia.

\section{REFERENCIAS}

[1] OCDE, “Perfilando la transformación digital en América Latina: Mayor productividad para una vida mejor, OECD Publishing" pp. 28, 2019. Disponible en: https://doi.org/10.1787/4817d61b-es
[2] Almaraz Menéndez, F., Maz Machado, A., \& López Esteban, C., Análisis de la transformación digital de las Instituciones de Educación Superior. Un marco de referencia teórico". EDMETIC, "6(1), 181, pp 189, 2016. Disponible en: https://doi.org/10.21071/edmetic. v6i1.5814

[3] Dening, P., Computer Science: The Discipline. Encyclopedia of Computer Science, 1999.

[4] Serna, E., "La abstracción como componente crítico de la formación en ciencias computacionales". Revista Avances en Sistemas e Informática. Vol 18, pp. 80.

[5] Wauters, R. "Sequoia Invests \$8 Million In Messaging App Maker WhatsApp: Sources. Techcrunch", 2001. Disponible en https:// techcrunch.com/2011/04/08/sequoia-whatsapp-funding/

[6] Huertas, D., "Unos 20 millones de colombianos no tienen acceso a Internet". RCN Radio, 2019. Disponible en https://www.rcnradio. com/tecnologia/unos-20-millones-de-colombianos-no-tienen-acceso-internet

[7] DANE, "Boletín Técnico Indicadores básicos de tenencia y uso de tecnologías de la información y comunicación - TIC en hogares y personas de 5 y más años de edad”, 2018. Disponible en https://www.dane.gov.co/ files/investigaciones/boletines/tic/bol_tic_ hogares_2018.pdf

[8] Bartolomé Pina A., Bellver Torlà C., Castañeda Quintero L., Segura A, "Blockchain en educación: introducción y crítica al estado de la cuestión". EDUTEC. Revista Electrónica de Tecnología Educativa número 61. ISSN 1135-9250, pp. 6, 2017. Disponible en: https: / / www.edutec.es/revista/index.php/ edutec-e/article/view/915

[9] Jaap T., "Desarrollo del liderazgo". Traducción Zulma Ramos Ramírez. Serie empresarial Fondo Editorial Legis (c) 1991, Bogotá, Colombia, pp. 6, 1989.

[10] Drucker P., "Gerencia para el futuro. El decenio de los 90 y más allá". Bogotá, Colombia: Grupo Editorial Norma, pp.118, 1992. 
[11] Maldonado Mera, B., Buenaño Cabrera, J., Benavides Espinosa K. "Aproximación a un modelo de gobernanza en las universidades públicas de la provincia de Pichincha del Ecuador", Revista Científica "Visión de Futuro", Universidad Nacional de Misiones, Argentina, vol. 23, núm. 2, s.p, 2019. Disponible en: http://www.redalyc.org/articulo. oa?id=357960138007

[12] Maldonado Mera, B., Buenaño Cabrera, J., Benavides Espinosa K. “Aproximación a un modelo de gobernanza en las universidades públicas de la provincia de Pichincha del Ecuador", Revista Científica "Visión de Futuro", Universidad Nacional de Misiones,
Argentina, vol. 23, núm. 2, s.p, 2019. Disponible en: http://www.redalyc.org/articulo. oa?id=357960138007

[13] Westerman, G. MIR Sloan Executive Educatios Blog. Redefining the digital divide. [Blog post], 2014. Disponible en: https://executive.mit.edu/blog/redefining-the-digital-divide\#.VDx4yhaOOAg

[14] Morales Arce, Víctor Gerardo, “Desarrollo de competencias digitales docentes en la educación básica". ISSN: 1665-6180, pp. 91, 2013. Disponible en: https://www.redalyc. org $/$ articulo.oa?id=688/68830443008 